\title{
Clinical, dermoscopic and confocal features of nevi and melanomas in a multiple primary melanoma patient with the MITF p.E318K homozygous mutation
}

\author{
Bassoli $\mathrm{S}^{1}$, Pellegrini $\mathrm{C}^{2}$, Longo $\mathrm{C}^{1,3}$, Di Nardo $\mathrm{L}^{2}$, Farnetani $\mathrm{F}^{1}$, Pellacani $\mathrm{G}^{1}$, Fargnoli $\mathrm{MC}^{2}$
}

Dermatology and Venereology, University of Modena and Reggio Emilia, Italy; ${ }^{2}$ Biotechnological and Applied Clinical Sciences Department, University of L'Aquila, Italy; ${ }^{3}$ IRCCS Arcispedale Santa Maria Nuova, Reggio Emilia, Italy

\section{INTRODLCTION}

The MITF gene is an oncogenic melanocyte lineage-specific transcription actor involved in cell survival differentiation and proliferation ${ }^{1}$. The p.E318K variant has been risk, development of multiple melanom risk, development of multiple melanom (MPM) and high nevus count $2,3,4,6,6$. We report the first case of the MITF p.E318K melanoma.

\section{MATERLALS AND METHODS}

A man was referred to our Clinic for a routine follow-up visit because of a personal history of Germline DNA was extracted from whole blood by standard procedures using QIAamp three primary melanomas, all located on the back, and a high number of melanocytic nevi DNA Blood kit (Qiagen, Hilden, Germany).

(total nevi count $>250$ ). Dermoscopic (DerLite Photo ${ }^{\otimes}, 3 \mathrm{Gen}$, San Juan Capistrano, Germline mutations of exons $1 \alpha, 1 \beta, 2$ e 3 including the exon-intron boundaries of California) and confocal (Vivascope ${ }^{\circledR} 1500$, MAVIG, Germany) images were available for CDKN2A, exon 2 of $C D K 4$, the entire open reading frame of $M C I R$ and exon 10 of $M I T F$ two out of three melanomas and for several patient's melanocytic nevi.

We describe the phenotypic characteristics of the patient and the dermoscopic and confocal Genetic Analyzer 3500 (ThermoFisher Scientific, CA). The p.S270N POTI mutation was features of his melanomas and melanocytic nevi.

\section{RESULIS}

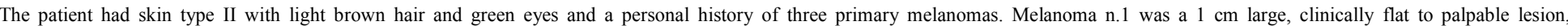

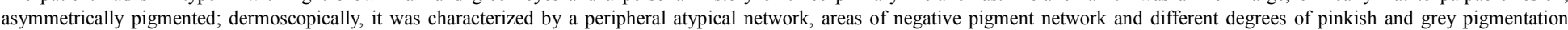

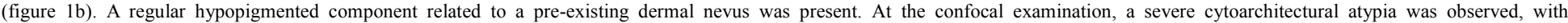

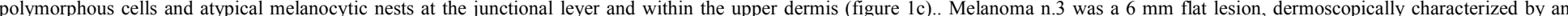

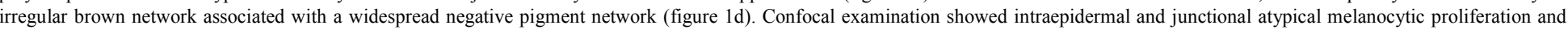

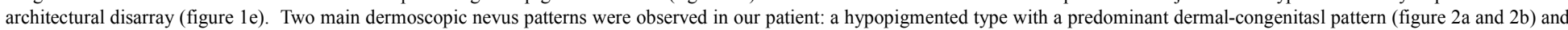

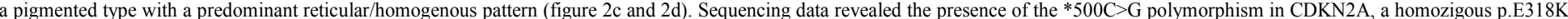
mutation in MITF (figure 3) and the p.R160W R allelic variant in MC1R, while no melanoma predisposing mutations were found in CDK4 and POT1 genes.
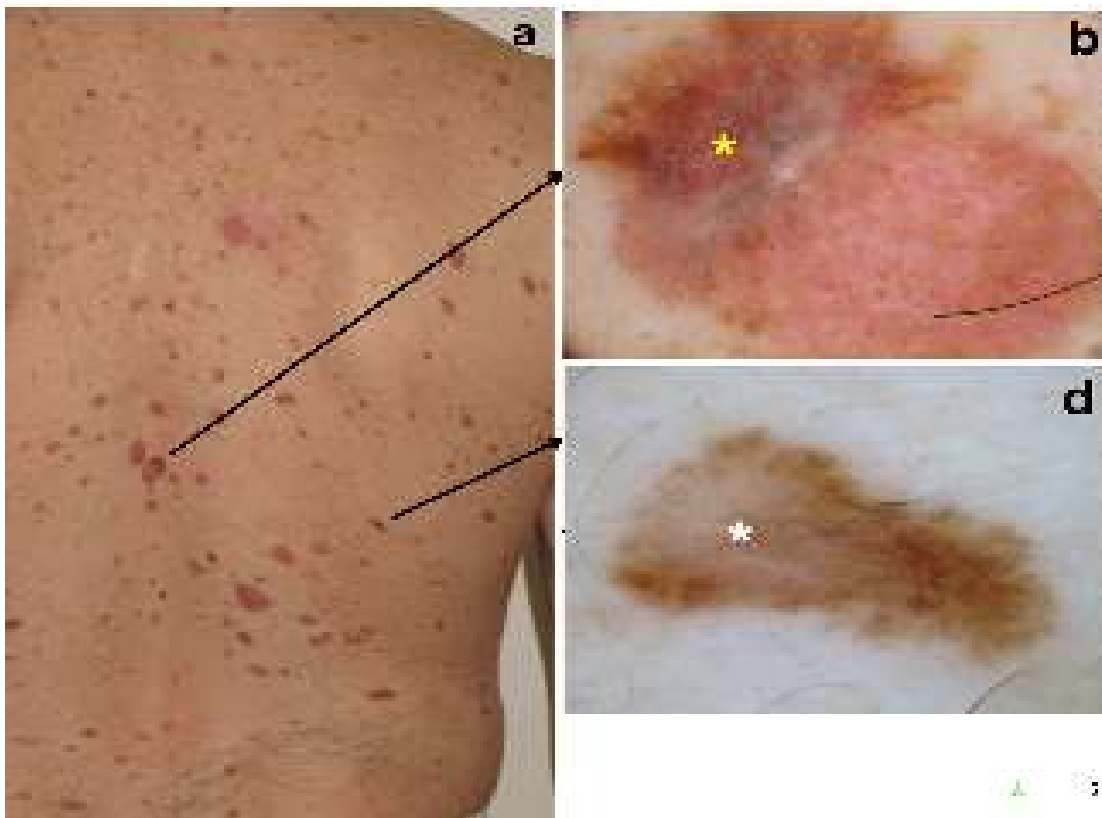
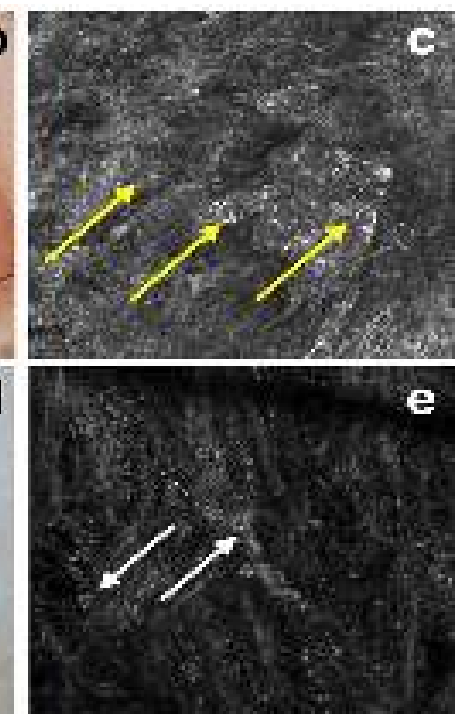

Figura 1. (a) Clinical image of the back of a 48 year-old male patient. Arrows indicate two of the three melanomas; (b) Dermoscopic image of Melanoma n.1. Strongly asymmetrical structure and dermoscopic islands are the main dermoscopic features; (c)Confocal image at the lower junctional layer of the dermoscopic island of the lesion in $1 \mathrm{~b}$ showing thickned atypical meshwork with numerous atypical nests at the junction and upper dermal layer (yellow arrows); (d) Asymmetrical distribution of dermoscopic features of melanom $n$ Areas of stypical network associated with. Areas of stypical network associated with a negative pigment network area (white star); (e) Confocal image at the junctional layer of the lesion in $1 \mathrm{~d}$ showing an atypical meshwork with numerous atypical nucleated cells (white arrows).

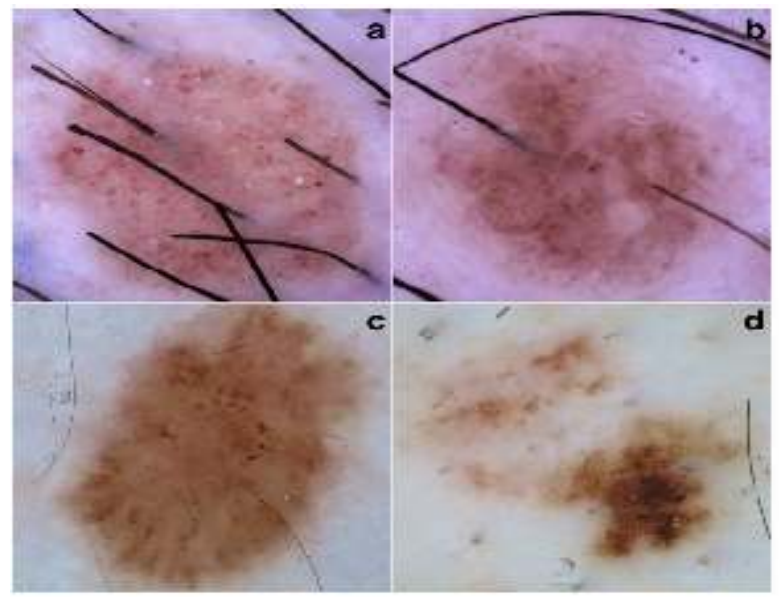

Figura2. a-b) Hypopigmented nevus with a predominant dermal-congenital pattern: globular/homogenous with a pink to light-brown pigmentation and numerous comma vessels. c-d) Pigmented nevus, with a predominant reticular/homogenous pattern:a brown pigmented network, associated with areas of homogeneous brownpigmentation or follicular hypopigmentation with regularly distributed globules, as observed in acquired nevi of the patient. 